\title{
Bleeding of a familial cerebral cavernous malformation after prophylactic anticoagulation therapy
}

\author{
Case report
}

\author{
Eugenio Pozzati, M.D., Mino Zucchelli, M.D., Anna Federica Marliani, M.D., \\ ANd Luca Albini Riccioli, M.D.
}

Departments of Neurosurgery and Neuroradiology, Bellaria Hospital, Bologna, Italy

\begin{abstract}
$\checkmark$ The risk of hemorrhage in cavernous malformations (CMs) depends on lesion, host, and environmental factors. Anticoagulation therapy is a well-known risk factor for intracerebral bleeding, but the occurrence of hemorrhages in patients with CMs has not been reported. Low molecular weight heparin therapy is generally considered to be safe, although significant hemorrhagic complications have recently been reported. The authors report a case of intralesional bleeding in a CM after prophylactic anticoagulation therapy was administered in a patient with the familial form of the disease. The leaky endothelial structure of CMs may constitute an unexpected target of the vascular effects of heparin.
\end{abstract}

\section{KEY WORDS - cavernous malformation - heparin • anticoagulation therapy • cerebral hemorrhage}

$\mathrm{C}$ AVERNOUS malformations consist of vascular sinusoids lined by endothelium embedded in a collagen matrix. Cerebral CMs may present with headache, seizures, mass effect, and hemorrhage. They exist in two forms, sporadic and familial, and their hemorrhagic potential is variable and depends on several host and lesion factors. The risk of hemorrhage in patients with CMs who undergo procedures requiring anticoagulation therapy is unknown. We present the case of a young woman with familial CMs who underwent a hysterectomy followed by anticoagulation therapy with LMWH for 1 week. An MR image obtained after 20 days demonstrated a previously intact $\mathrm{CM}$ with subacute hemorrhage corresponding to the period of heparin therapy. The primarily endothelial structure of CMs may be associated with a higher risk of bleeding due to the peculiar effects of heparin on the endothelial cells.

\section{Case Report}

History and Examination. This 42-year-old woman initially presented with epileptic seizures secondary to multiple cerebral CMs, the largest of which, a hemorrhagic lesion in the right frontal lobe, was excised in 1999. Her son had undergone surgery at 4 years of age for a cerebel-

Abbreviations used in this paper: $\mathrm{CM}=$ cavernous malformation; $\mathrm{LMWH}=$ low molecular weight heparin; $\mathrm{MR}=$ magnetic resonance; $\mathrm{tPA}=$ tissue plasminogen activator. lar CM, and three brothers and sisters as well as other relatives were all affected by CMs. She also had retinal and skin CMs. She was found to harbor a mutation in the KRIT1 gene at the CCM1 locus, but unfortunately we do not know the precise genetic sequence. Routine MR imaging in April 2004 showed stable multiple CMs without recent hemorrhage (Fig. 1).

Operation and Postoperative Course. On October 7, 2005 , the patient underwent a hysterectomy for fibromatosis, and postoperative LMWH (nadroparin 4000 IU daily) was administered for 8 days. At the end of treatment she reported dysesthesia of the right hand. Her symptoms gradually subsided, and an MR image obtained on November 8, 2005, revealed a subacute hemorrhage and perifocal edema corresponding to a left parietal CM, which had previously been stable. The MR imaging characteristics were consistent with bleeding that had occurred during or immediately after the postoperative anticoagulation therapy (Fig. 2). In particular, the signal intensity of the blood on the $\mathrm{T}_{1}$ - and $\mathrm{T}_{2}$-weighted images was high, corresponding to extracellular methemoglobin and to a hematoma in the late subacute phase (1-4 weeks). A follow-up MR image obtained in December 2005 showed total resorption of the hemorrhage.

\section{Discussion}

The basic vascular element of CMs consists of leaky endothelium-lined sinusoids. No tight junctions exist at endothelial interfaces, and gaps between endothelial cell 

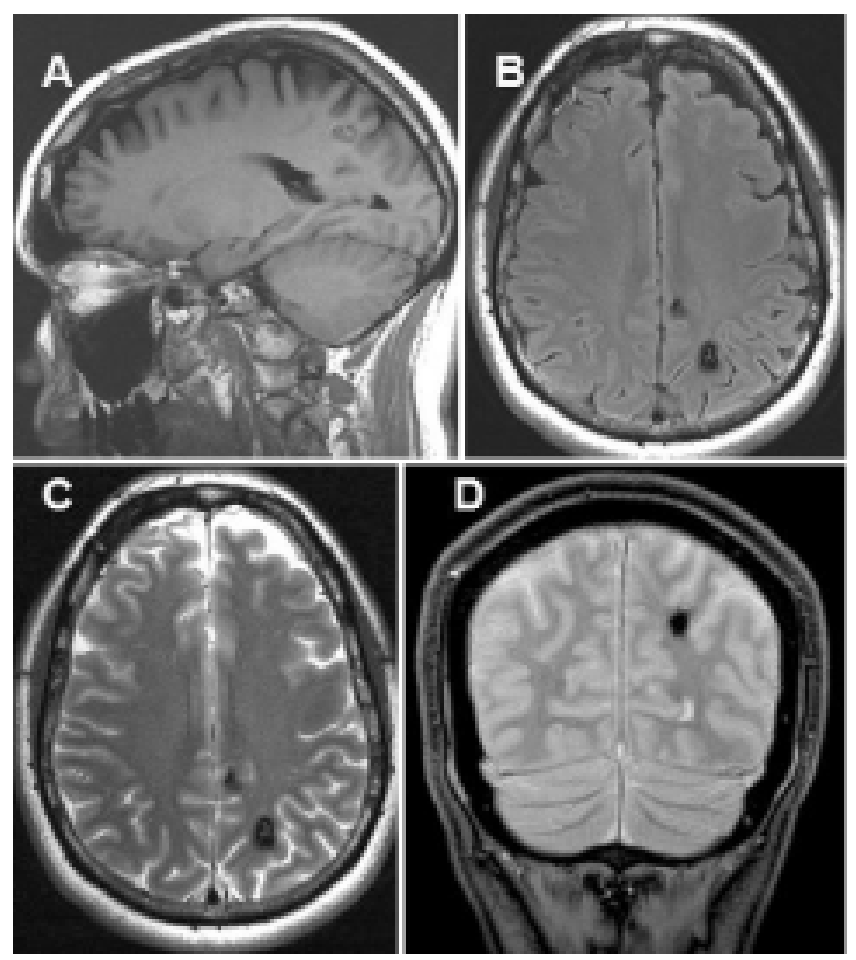

FIG. 1. Cerebral MR images obtained in April, 2004. Sagittal spin echo $\mathrm{T}_{1}$-weighted image $(\mathrm{A})$ revealing a lesion that is isointense to white matter, with a hypointense rim. Axial fluid-attenuated inversion-recovery $\mathrm{T}_{2}$-weighted $(\mathrm{B})$ and fast-recovery fast spin echo $\mathrm{T}_{2}$-weighted $(\mathrm{C})$ images demonstrating the typical "reticulated" appearance of a cavernous angioma. Coronal $\mathrm{T}_{2} *$-weighted image (D), with a prominent low signal resulting from the gradient echo effect of hemosiderin.

processes may expose the basal lamina, leading to a defective blood-brain barrier: leakage of red blood cells toward the surrounding parenchyma constitutes the basic pathological mechanism of CMs. ${ }^{2}$ Furthermore, major bleeding may occur both extra- and intralesionally: the risk of overt hemorrhage in CMs depends on the patient's age and sex and on factors related to the lesion (location, associated venous malformation, previous bleeding). ${ }^{10}$ In addition, environmental factors (radiation therapy, infection, and hormone therapy) ) $^{4,6,11}$ may interfere with the biological behavior of CMs.

Anticoagulation therapy constitutes a precise risk factor for intracerebral bleeding. Prophylactic anticoagulation therapy with LMWH is performed in a vast number of patients, with a very low incidence of hemorrhage. In particular, the occurrence of hemorrhage in CMs has not been precisely reported. The CMs exist in two forms, familial and sporadic, each with a different hemorrhagic potential: the familial form represents a variable percentage ranging from 24 to $56 \%$ of cases. ${ }^{7}$ Familial CMs are linked to mutations of the KRIT1 gene at the CCM1 locus and to mutations at two other loci, CCM2 and CCM3. The KRIT1 gene probably plays a part in the formation of endothelial cell junctions ${ }^{2}$ and contributes to the "incompetency" of the capillary sinusoids constituting the structure of CMs.

The proportion of patients with symptomatic familial CMs who have cerebral hemorrhages is higher than that

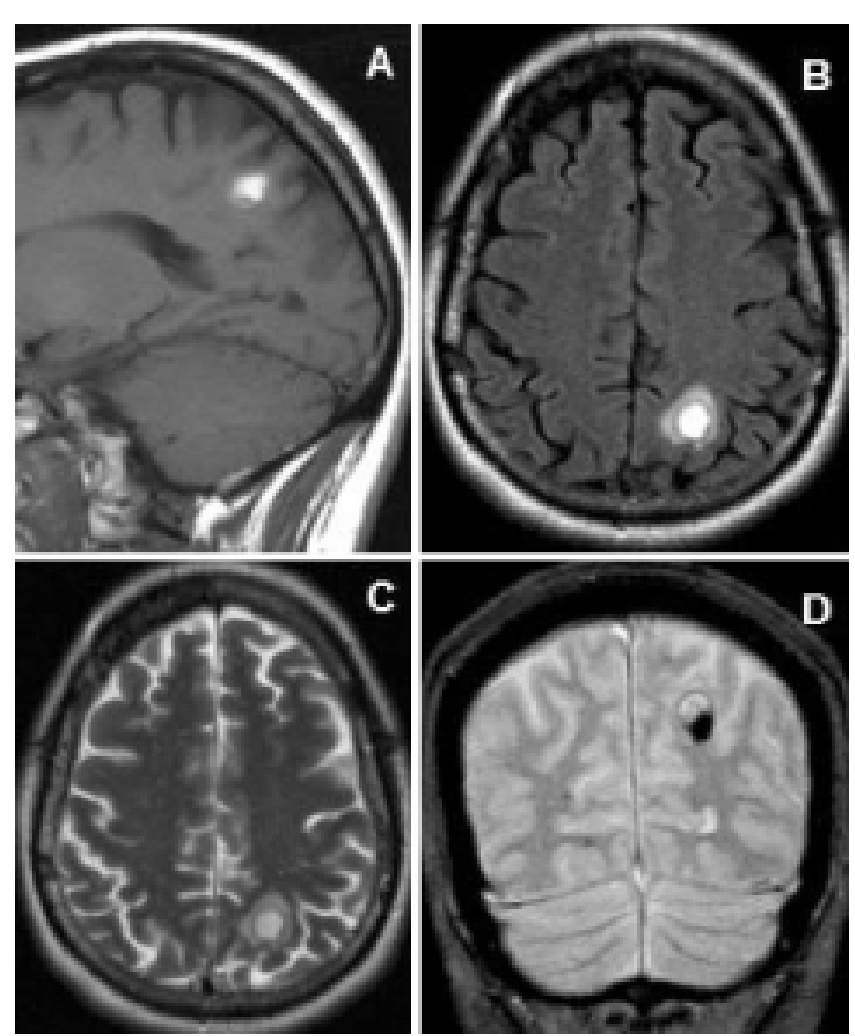

FIG. 2. Cerebral MR images obtained on November 8, 2005, 1 month after the operation and 20 days after anticoagulation therapy. Sagittal spin echo $\mathrm{T}_{1}$-weighted (A), axial fluid-attenuated inversion-recovery $\mathrm{T}_{2}$-weighted (B), and fast-recovery fast spin echo $\mathrm{T}_{2^{-}}$ weighted $(\mathrm{C})$ images demonstrating a round hematoma surrounded by edema corresponding to the previous unruptured cavernoma. On a coronal $\mathrm{T}_{2}{ }^{*}$-weighted (D) image, the lesion has two components: superior, which is hyperintense due to the blood, and inferior, which is hypointense due to the persistence of the hemosiderin.

reported for the sporadic forms. ${ }^{7}$ The familial form is at higher risk of bleeding, which is related both to the number of lesions present in an individual and to the genetic derangement on the ultrastructure of the endothelial wall. Familial CMs of the central nervous system and retina are recognized as an autosomal-dominant disorder and are symptomatic in a large number of cases $(\sim 70 \%) .{ }^{3}$ A novel KRIT1/CCM1 truncating mutation seems to be specifically associated with the occurrence of cerebral and retinal CMs. ${ }^{8}$

Similarly, a true oculoneurocutaneous syndrome or phacomatosis (as in our case) with additional skin cavernomas related to a specific mutation has been reported, ${ }^{12}$ indicating that a careful correlation of genotype and phenotype may help in predicting disease behavior and bleeding potential.

In our case, the hyperintensity of the lesion on $\mathrm{T}_{1^{-}}$and $\mathrm{T}_{2}$-weighted images indicates a hematoma in the late subacute phase, corresponding to extracellular methemoglobin formed between 1 and 4 weeks after the bleeding, which presumably occurred at the end of the prophylactic anticoagulation therapy. In addition, the presence of perifocal edema indicated a recent hemorrhage in the same time interval. 


\section{Bleeding of a familial cerebral CM after anticoagulation therapy}

The origin of the hemorrhages that occur after anticoagulation therapy in CMs is probably related to its effects on the vascular endothelium, which is one of the sites of action for heparin. ${ }^{9}$ In one study, CMs contained endothelial cells that stained for tPA, supporting the hypothesis that a local thrombolytic process may be responsible for the hemorrhagic nature of CMs. ${ }^{5}$ The tPA is expressed in a number of different cell types in the developing nervous system: permanent endothelial cell levels of tPA in CMs may indicate that they are a holdover from the primitive developing brain. ${ }^{1}$ It is known that heparin treatment may increase tPA activity ${ }^{13}$ and, as a consequence, may promote hemorrhage in CMs.

\section{Conclusions}

Familial CMs are at major risk of bleeding due to specific genetic abnormalities that translate into incompetent endothelial vessels and possibly increased sensitivity to the effects of heparin, and that should be added to the "environmental" factors contributing to the bleeding of CMs. We recommend caution in the use of prophylactic anticoagulation therapy in patients with familial CMs, but formal contraindications to heparin in the broad setting of CMs should be confirmed by additional observations.

\section{References}

1. Carmel PW: Editorial Comment. Pediatr Neurosurgery 25: 137-142, 1996 (unverified)

2. Clatterbuck RE, Eberhart CG, Crain BJ, Rigamonti D: Ultrastructural and immunocytochemical evidence that an incompetent blood-brain barrier is related to the pathophysiology of cavernous malformations. J Neurol Neurosurg Psychiatry 71: 188-192, 2001

3. Dobyns WB, Michels VV, Groover RV, Mokri B, Trautman JC, Forbes GS, et al: Familial cavernous malformations of the central nervous system and retina. Ann Neurol 21:578-583, 1987

4. Fender LJ, Lenthall RK, Jaspan T: De novo development of presumed cavernomas following resolution of $E$. Coli subdural empyemas. Neuroradiology 42:778-780, 2000

5. Frim DM, Zec N, Golden J, Scott RM: Immunohistochemically identifiable tissue plasminogen activator in cavernous angioma: mechanism for re-hemorrhage and lesion growth. Pediatr Neurosurg 25:137-142, 1996

6. Gazzaz M, Sichez J, Capelle L, Fohanno D: [Recurrent bleeding of thalamic cavernous angioma under hormonal treatment. A case report.] Neurochirurgie 45:413-416, 1999 (Fr)

7. Labauge $P$, Laberge S, Brunereau L, Levy C, Tournier-Lasserve E, the Société Francaise: Hereditary cerebral cavernous angiomas: clinical and genetic features in 57 French families. Lancet 352:1892-1897, 1998

8. Laberge-Le Couteulx S, Brezin A, Fontane B, Tournier-Lasserve E, Labauge P: A novel KRITI/CCMI truncating mutation in a patient with cerebral and retinal cavernous angiomas. Arch Ophthalmol 120:217-218, 2002

9. Nader HB, Lopes CC, Rocha HAO, Santos EA, Dietrich CP: Heparins and heparinoids: occurrence, structure and mechanism of antithrombotic and hemorrhagic activities. Curr Pharm Des 10:951-966, 2004

10. Pozzati E, Acciarri N, Tognetti F, Marliani F, Gianspero F: Growth, subsequent bleeding, and de novo appearance of cerebral cavernous angiomas. Neurosurgery 38:662-670, 1996

11. Pozzati E, Giangaspero F, Marliani F, Acciarri N: Occult cerebrovascular malformations after irradiation. Neurosurgery 39: 677-682, 1996

12. Sarraf D, Payne AM, Kitchen ND, Sehmi KS, Downes SM, Bird AC: Familial cavernous hemangioma: an expanding ocular spectrum. Arch Ophthalmol 118:969-973, 2000

13. Zhao BQ, Ikeda Y, Ihara H, Urano T, Fan WY, Mikawa S: Essential role of endogenous tissue plasminogen activator through matrix metalloproteinase 9 induction and expression on heparin-produced cerebral hemorrhage after cerebral ischemia in mice. Blood 103:2610-2616, 2004

Manuscript received May 15, 2006.

Accepted in final form June 1, 2006.

Address reprint requests to: Eugenio Pozzati, M.D, Divisione di Neurochirurgia, Ospedale Bellaria, Via Altura 3, 40139 Bologna, Italy. email: Eugenio.Pozzati@ausl.bo.it. 